\title{
Delivering Sexual and Reproductive Health Education to Girls
}

\author{
Are Helplines Useful?
}

Joan Njagi

$\cos$

\begin{abstract}
The use of helplines to deliver sexual and reproductive health (SRH) education to girls seeking such information and services can break down barriers created by low access and top-down approaches. However, it is important to interrogate their effectiveness in addressing the SRH needs of girls, particularly in contexts in which hierarchical social relations prevail and conservative religious and cultural norms dictate appropriate expressions and experiences of sexuality for girls and young women. In this article I use data drawn from a qualitative case study of a children's helpline in Kenya to interrogate the interplay of power and culture in the delivery of SRH information to girls. The findings reveal that while this particular communication technology presents, potentially, a revolution in such delivery, power dynamics and cultural norms still pose barriers.
\end{abstract}

Keywords: agency, communication technologies, culture, girls' sexuality, participation, power, sexuality, voice

Communication technologies such as social and mobile media are considered useful in contributing to breaking down barriers of access to mainstream media platforms such as TV, radio, and newspapers, thereby increasing social and political representation. They can transcend infrastructural barriers to amplify the voices of girls and young women in challenging social norms that marginalize and exclude them, and define their agenda. They can influence social norms and public policies, even in rural and under-resourced communities with limited access to technology (Hart and Mitchell 2015; The Women's Rights Association Programme for Progressive Communication (WRPAPC) 2012). Making reference to the internet, for instance, WRPAPC (2012) argues that communication technologies create avenues for negotiation and the realization of the rights of people with little access to the public sphere because of the intersection of multiple forms of discrimination including gender, age, class, and/or sexuality.

The positive view of communication technologies reflects the progressive shift from their being viewed only as agents of harm leading to cyber-bullying 
and as being responsible for increasing children's access to pornography, for example, to being recognized as important to the enhancement of voice and agency. However, discussing communication technologies solely from negative or positive points of view, in failing to demonstrate the nuances involved, offers limited understanding of their limits and potential.

In this article, based on my Masters dissertation research, I use the case of a children's helpline in Kenya to interrogate the extent to which the helpline as a form of communication technology is being used to address girls' SRH needs and lived realities effectively. Given the hierarchical nature of adult-child relations in conservative Kenyan society, where religious and cultural values that promote heteronormativity and gender inequality and deny the validity of girls' sexuality are deeply embedded, I examine the interplay of culture and power dynamics in the use of the helpline particularly with reference to girls' sexuality.

\section{The Potential of Helplines to Enhance Child Protection and SRH Rights}

Children are among the most vulnerable members of society given the low status accorded to them because of their age and perceived powerlessness. In addition to this, other group-based disadvantages such as gender, class, and (in)ability intersect to increase the vulnerability of girls in particular, exposing them to physical, emotional, sexual, and structural violence and abuse. Studies indicate that up to 50 percent or more of girls in Kenya have experienced different forms of sexual abuse ranging from sexual harassment that includes inappropriate touching, declarations of love from adults including teachers, and unwanted sex (Ruto 2009).

Reporting violence and abuse can pose challenges for girls because the people abusing them-often parents, teachers, and relatives-are also charged with their protection (Childline Kenya (CLK) 2013a). Sometimes the abusers threaten girls with reprisals if they report this abuse; reporting poses a greater challenge for girls in contexts in which practices that violate their rights are socially and culturally accepted. Even when girls report sexual abuse, their protection may not be guaranteed since their caregivers sometimes disbelieve them or opt to conceal the abuse to protect the family so as to hold it together. In schools, girls are discouraged from reporting sexual abuse because teachers often fail to take them seriously, and, in some cases, these girls are accused of having sent signals that encourage abuse (Pattman 
and Chege 2003). Avenues for reporting abuse outside the home or school are either out of reach of children, intimidating, or not child-friendly. Moreover, the taboos surrounding the discussion of sex makes it even more difficult for girls to seek SRH information or report sexual violence. Deevia Bhana (2008) argues that the eradication of traditional African sex education structures has generated different and uneven views about sex, and has introduced the concept of shame, particularly for girls. As Bernard Njau et al. (2007) have pointed out, this leaves many young people unable to find information to help them deal appropriately with sexuality and, as Carolyne Njue et al. (2011), have observed, while they are not completely ignorant of SRH issues, they often lack appropriate avenues and opportunities to confirm what they have heard, or ask questions about SRH that concern them.

The regulation of girls' sexuality not only restricts and limits what girls can say or do, it also increases their vulnerability in relationships. The dichotomous construction of girls' sexuality as good or bad, moral or immoral constrains girls from openly discussing their sexuality and their concerns related to sex as well as from reporting sexual abuse (Pattman and Chege 2003).

Given the traditional cultural and religious norms in Kenya, SRH information tends to be gender-biased; this poses an even greater challenge for girls and young women, in addition to promoting heteronormative ideals, thereby excluding young people with non-conforming sexual identities. Available SRH information fails to address the varied lived realities of girls' sexuality by promoting abstinence and other homogeneous and un-contexualized strategies such as the $\mathrm{ABC}$ strategy (Abstain, Be faithful, Condomize) to prevent HIV infection.

Given advances in technology, the situation is changing and girls no longer have to suffer in silence. As Mirkka Mattila (2011) reminds us, technology has provided ways of breaking the silence about child abuse and enhancing the protection of girls and children in general. Helplines, hotlines, and short messaging platforms are now accessible to children and also to adults concerned with the welfare of children. These are sometimes free of cost and often operate 24 hours a day. Helplines are commonly used to report female genital mutilation, child marriages, sexual harassment and abuse, and their being trafficked for domestic labor among other rights violations commonly experienced by girls (see also CLK 2014). Data from these helplines provides information on specific child protection issues affecting girls and this, in turn, can drive further research, outreach, and advocacy so as to influence policy (Mattila 2011). 
Helplines provide safe spaces in which girls can discuss issues affecting them with trained counselors while being assured of confidentiality and anonymity. They also provide girls with a platform from which to voice their concerns and exercise agency in seeking SRH information free from the influence of cultural and religious norms. Although helplines are breaking new ground in protecting children and providing avenues for them to access SRH information, allowing them to exercise agency and potentially chart their agenda, there is a need to question whether these approaches do indeed break down the barriers experienced by girls in trying to access SRH information through conventional channels.

\section{Questioning the Democratic and Participatory Nature of Helplines}

The construction of children as holding rights and as being active agents is common to many development programs. As Jason Hart (2008) has explained, participation in these programs is perceived to transform relationships between adults and children so as to realize children's rights. Children's participation, which is often cast within the frameworks of agency and rights-based approaches, and girls' empowerment are thus lauded as vital enablers of voice and choice, particularly for girls who suffer multiple forms of oppression because of their age, sex and gender, and (sometimes) economic situation. The empowerment of girls through participation in development initiatives is considered critical for inclusive decision-making.

The promotion of girls' empowerment and children's agency in development has, however, been critiqued as a neoliberalist discourse that focuses on individual empowerment to legitimize a neoliberal development agenda (Bent 2013; Bordonaro 2012; Cornwall and Brock 2005). The practices related to girls' empowerment and children's participation in development initiatives often fail to challenge existing forms of power, and, in some cases, it becomes the means through which adult power is entrenched (Bent 2013; White and Choudhury 2007). This may take the form of programs being more concerned with molding girls to meet social expectations rather than working towards social transformation. Moreover, the simplistic promotion of children's agency and girls' empowerment may fail to acknowledge that participation involves power that is relational and situational. As Emily Bent (2013) has made clear, the simplistic promotion of girls' agency and empowerment ignores the ways in which socio-cultural power structures influence their lives. 
The participation of girls in public spheres needs to be examined as one involving power, but also as one that is influenced by culture. Lorenzo Bordonaro (2012) argues that recognition of children's agency does not necessarily expand children's freedom for increased participation in society because of the unresolved tensions between the idea of children's agency on the one hand, and the local cultural structures and disciplinary interventions on the other that constrain such agency. This is more pronounced for girls and their sexuality because their lives are more regulated than are boys' lives. Therefore, while development initiatives may provide room for girls to participate, their agency is prescribed by the culture in which they exist, and must be understood from a political and social point of view that is connected to local assumptions about gender and the rights and freedoms of girls and young women (Bordonaro 2012).

While helplines have been constructed as tools for securing democratic space, removing participation barriers, and allowing girls to exercise their agency and drive their agendas, it is important to interrogate the reality of this perceived notion of democracy. Casey Burkholder et al. (2015) argue that as spaces provided by communication technologies become more accessible, thereby increasing voice and participation, power and how it operates in these spaces remains inadequately discussed. They further argue that even in such spaces power dynamics can intentionally or unintentionally alter and/or transform the voices of marginalized groups in their attempts to effect social change.

Discussions on the tendency of spaces provided by communication technologies to be undemocratic often focus on the internet and on the influence of content and freedom of information by governments, private entities, and other actors with existing power structures, driven by the need to regulate and preserve gender and sexual norms (see WRPAPC 2012). However, these discussions focus on the often evident and even expected regulation of the internet and fail to consider that such regulation can be subtle, almost invisible, unexpected, and sometimes unintentional, and can extend to communication technologies that are not necessarily internet supported such as helplines. In this article, I explore how both overt and covert public policies, culture and tradition, rules and norms and other conditions can alter the voices of girls expressing their concerns, particularly in relation to SRH, thereby constraining their agency in spaces provided by helplines.

In the following sections I examine the interplay of culture and power that covertly regulates girls' sexuality and constrains their agency, thereby inhibiting the ability of helplines to promote voice and agency. 


\section{Methodology}

This article is based on a case study of CLK, a Kenyan organization that promotes children's rights and that operates a 24-hour national toll-free helpline, in conjunction with the Government of Kenya through the ministry of Gender, Children and Social Development's Department of Children's Services. "Through its trained telephone counselors, [CLK] provides high-quality personalized services to children, giving them the opportunity to report abuse, receive counseling and make informed choices" (Stars Foundation 2010: n.p.). CLK also works with a well-established network of referral partners to offer support to children and their families through counseling, legal advice and representation, child abuse investigations, home visits, and public education on child rights and parenting skills for the prevention of abuse (Mattila 2011).

The study used qualitative techniques including in-depth semi-structured interviews with six staff members including three senior officials and three randomly selected outreach workers/counselors. In addition, I reviewed CLK's data and reports from 2012 to 2015. Information about the girls in the study was obtained from sampled cases in CLK's reports. All sampled cases involved girls below the age of 18 either directly, where the girl was the caller, or indirectly, where another child or an adult called on behalf of a girl.

\section{Ethical Concerns}

Before embarking on the research, I obtained ethical clearance from the Institute of Education (IOE), University College London (UCL), and obtained consent from research respondents. I also signed CLK's child protection and data confidentiality policies. There was no direct contact with the children or caregivers who called the helpline since CLK reports and interviews provided sufficient information on how cases were handled.

In line with counseling ethics, counseling is offered in confidentiality, which can be breached only in situations in which the caller is in danger or poses a danger to others. CLK further observes strict measures to maintain confidentiality and the anonymity of the callers such as ensuring that information about the callers is not accessible to the public. In instances where information is made public, the identity of callers is concealed. These measures ensured that children were not exposed to harm during the study. 


\section{Findings}

\section{Girl's Agency Coexisting with Adult Imposition}

The use of helplines to deliver SRH information is indeed revolutionary, with the potential to promote the agency of girls to chart their own agendas. Similar to other forms of communication technologies, helplines can also enhance children's participation, which is not only considered a right, but also a facilitator of other rights (Bhana 2007a). Unlike conventional forms such as school-based SRH education delivery, which have been critiqued for using top-down approaches (Kendall and O'Gara 2007), helplines provide a bottom-up approach allowing young people to seek out services and share their concerns. This is particularly critical for girls who are most affected by negative SRH outcomes.

The importance of helplines in promoting the participation of children and advancing the agency of girls is clearly demonstrated by CLK. Between January and June 2014, CLK received 476 SRH related cases from 45 of the 47 counties in Kenya. The cases included 4 on commercial child sex work, 68 on early and forced marriages, 9 on masturbation and pornography, 95 on opposite sex relationships, 38 on reproductive health, 248 on sexual abuse, 10 related to HIV and AIDS, and 3 on sexual identity. Of the 476 cases, almost 80 percent (366 in total) of the calls were received from girls (CLK 2014).

It is important to note that even though boys generally make more calls to the helpline compared to girls, girls called more often than boys to discuss SRH issues and to report sexual abuse and commercial exploitation (CLK 2013a, 2013b, 2014, 2015). This may be driven by the fact that girls are more vulnerable to sexual abuse and negative SRH outcomes.

Moreover, given the tendency of social constructions of girls' sexuality to silence them from discussing SRH issues openly, a confidential and anonymous helpline may have high appeal for girls. Rob Pattman and Fatuma Chege (2003) noted in their observations of life skills and HIV education in schools in East and Southern Africa that boys discussed their sexuality and sexual experiences openly and explicitly, while girls had to be careful about expressing sexual desire or experience for fear of being labelled bad. This construction of girls' sexuality has obvious implications for voice and also increases their vulnerability in opposite sex relationships.

The cases presented to CLK by girls demonstrate the power of helplines to give voice to marginalized groups. As I discuss in Njagi (2014), the actions taken by CLK as a result of this further demonstrate the potential of 
helplines to give power to them. CLK officers indicated that SRH issues and sexual abuse cases, which were often presented by girls, informed the design of a sexuality education program. The helpline thus played a key role in enhancing the agency of girls in presenting their SRH needs and in influencing the development of a program that would address their lived realities. However, while the SRH needs of girls were clearly demonstrated by their calls, this did not automatically translate into interventions to address the issues they raised. According to one interviewed staff member, the idea of developing a sexuality education program faced opposition because of personal perspectives within the team regarding children's sexuality. Some members of the team felt that a sexuality education program would encourage children to engage in sexual activity, and raise suspicion that organizations funding sexuality education programs were out to recruit children into nonconforming sexual identities. However, after a year-long consultative processes within the organization, sexuality education was added to CLK's outreach program.

Even with this success, the perspectives of adult implementers regarding children's sexuality overrode girls' SRH needs. The program is focused mainly on preventive and curative measures to deal with sexual abuse. However, even with the understanding that girls' engagement in sexual activities is not always a result of abuse, and that children can also engage in sex voluntarily or consensually, CLK's sexuality education is still focused on discussing the dangers of sex, with a clear stand that, as stated by one officer, "children should not engage in sex or sexual activity" (quoted in Njagi 2014: 52). This is particularly so for girls who bear the negative consequences of sexual activity more than boys. This perspective is held despite the fact that calls made through the helpline demonstrate sexual activity among girls, and in some cases by those who do not perceive sex as dangerous. The unilateral decision by CLK to use the helpline to promote abstinence, despite clear demonstration of sexual activity among girls and boys demonstrates how power and culture can constrain the agency of girls from being transformational, even in spaces provided by communication technologies such as the helpline.

\section{Pre-determined Content that Contradicts Children's SRH Needs}

Through the use of helplines, it is possible to understand the SRH needs and lived experiences of girls through firsthand information obtained from them. As Mattila (2011) reminds us, such information can be useful in designing relevant, child-centered programs and policies for girls. 
While the issues presented on the helpline demonstrate the need for a diverse range of SRH interventions for girls, the abstinence goal of the program prohibits them from having these discussions in an open manner. The response of one project worker demonstrates the predetermined goal to prevent and protect children from sexual activity. "In some cases, [success is achieved as] children vow not to engage in sexual activity, however, there isn't total conversion, this means that in some cases, children choose to continue being sexually active" (quoted in Njagi 2014: 63). Nel Van Beelen (2007) argues that many HIV prevention [and sexuality education] programs fail to be open and candid in discussing sex, sexuality, and sexual relationships, thus failing to address the needs of young people.

Higher usage of the helpline by girls on SRH issues, compared to boys also demonstrates that the availability of this confidential, child-centered avenue potentially offers a platform for girls to voice their concerns without social and cultural judgment on their expressing their sexuality. However, while the helpline presents an opportunity to address girls' SRH needs, it adopts the same moralistic, didactic, and authoritarian approaches used by their teachers in discussing sexuality and HIV- and AIDS-related issues (Chege 2007). This is evidenced by the abstinence approach that was adopted by all interviewed staff.

Similar to teachers' reasons, those of the CLK project staff for adopting approaches that discourage children's sexual activity included notions of morality and "the negative consequences on children's development" especially for girls who are at risk of pregnancy and procuring abortions. Sexual activities such as masturbation, touching, and kissing by children were discouraged since these "bring feelings which make children easy prey to sexual abuse by adults." As one project worker stated, "In case a child asked for a condom, we would not give condoms, but aim to change the mindset" or "help the child to set their priorities right" (quoted in Njagi 2014: 52).

Such an approach is detrimental to girls because it does not address the complexity of the SRH issues they present, and fails to recognize that girls engage in sexual activity for various reasons including engagement in transactional sex, which can also be coercive, to meet basic needs such as the purchase of sanitary towels (Ruto 2009). Moreover, abstinence-only programs fail to take into consideration the broadness and complexity of sexual activity.

At first glance this [abstinence] message seems simple and clear, but it is actually quite confusing. What is sexual activity? Is it only vaginal sex or is oral and anal sex included? For how long does one need to abstain? Is there room for slip-ups? 
Is abstinence realistic? Abstinence in itself is a limited message, it seems to be the only option and there is no room for mistakes. For many [girls] abstaining from sexual activity is not even a choice, for example when they are victims of sexual coercion and abuse. ... The abstinence message does not accept young people as sexual beings. (Youth Incentives 2008: 19)

The abstinence approach is not only guided by the socio-cultural norms on what counts as morality held by project workers, but also by Kenyan legal provisions that are guided by socio-cultural views of children's sexuality rather than girls' SRH realities. As stated by one project worker, "Our approach to sexuality education cannot contradict the law, which states that children cannot consent to sexual activity" (quoted in Njagi 2014: 53). Given the reality based on calls made to the helpline that girls are sexually active, and statistics on pregnancies, abortion, and HIV prevalence among young people, CLK is still not willing to use the data obtained from children to confront the law and stakeholders to revisit approaches to sexuality education since this will "not help children to change" but will instead "encourage children to have sex" and "create a sick society," (quoted in Njagi 2014: 57-58) according to interviewed project workers. Moreover, considering that CLK offers the helpline in conjunction with the Kenyan Government through the Department of Children Services, this poses a greater challenge for CLK to challenge laws that fail to acknowledge children's sexuality.

The approach taken by CLK and Kenyan law demonstrates the interaction between adult knowledge and power to perpetuate the discourse of innocence that constructs children, particularly girls, as asexual, lacking knowledge on sex and too young to know (Bhana (2007b, 2008). Bhana (2008) critiques the discourse of childhood's and girls' innocence as a Western construction that has been deployed by teachers to construct a singular girlhood that fails to recognize that African childhoods, especially those experienced by girls, are more diverse and unequal, and are experienced differently depending on race, class, and socio-economic indicators. Moreover, the discourse of innocence contradicts the lived realities of girls who may be surrounded by images of sex or who may be experiencing the effects of HIV and AIDS (Bhana 2007b).

\section{Varied and Complex SRH Needs Met with Narrow and Homogeneous Solutions}

Given that the helpline is nationally accessible at no cost, it is to be expected that the issues raised by girls and boys will be varied and complex. The following two sampled calls received by the helpline demonstrate these variations and complexities. 
A 16-year-old boy from Kitui sought advice through the helpline service. He had impregnated a 13-year-old class eight girl, and their respective families wanted him to take responsibility for his mistakes. While he was not opposed to this, his concern was that the girl wanted to procure an abortion. He was encouraged to raise the issue with his parents in the hope that there [would] be an agreement on how the child will be taken care of after birth. This would encourage the girl not to abort the baby. The option of the girl going back to school after delivery was also provided. Dangers of abortion were shared with him so that he has factual information to share with the girl and inform her decision. (CLK 2013b: n.p.)

A concerned mother calling from Kisumu reported that a form one [approximately 15 years of age] student at a nearby school had procured an abortion and was bleeding profusely. She said the girl's mother feared taking her to hospital. She was encouraged to explain to the mother the importance of taking the girl to hospital to avoid infections and complications. She was also encouraged to involve the police if necessary.

(CLK 2013b: n.p.)

However, while the issues presented demonstrate variation and complexity in the way they manifest and have an impact on the lives of girls, the responses demonstrate a homogeneous and one-size-fits-all approach, determined by a narrow and culturally prescribed conceptualization of children's sexuality.

Sexual activity refers to sexual intercourse between children of the opposite sex, as well as between a child and adult. It is unlawful, and a cause of psychological disorders. It also involves masturbation, pornography, sexual abuse and other sexual activities that could lead to sexual involvement. Children unwillingly or willingly engage in sexual activities without knowing the effects of these activities. We [CLK] are fighting against issues such as masturbation, which should not be allowed, because [it is] the genesis of sexual abuse. We inform children that friendships are not bad, but when they start to develop into sexual activity (including touching and kissing), such relationships at that tender age are wrong. We tell them that sexual activity has a negative impact on their health and other aspects of their lives including their careers. We tell [girls] that they could get pregnant and drop out of school and therefore need to concentrate on their education, up to masters' level. We give them information to enable them to make decisions, although we don't advocate for children to make a decision; a guardian must be involved to guide them positively. Children cannot consent. (quoted in Njagi 2014: 53)

Clearly, this definition of sexuality is far too narrow. The closest definition documented by CLK is that "sexuality category includes sex, gender identity and roles, sexual orientation, pleasure, intimacy and reproduction" (CLK 2012: n.p.).

The CLK definition of sexuality is simplistic compared to a more comprehensive definition offered by WHO (2006). 
Sexuality is a central aspect of being human throughout life and encompasses sex, gender identities and roles, sexual orientation, eroticism, pleasure, intimacy and reproduction. Sexuality is experienced and expressed in thoughts, fantasies, desires, beliefs, attitudes, values, behaviours, practices, roles and relationships. While sexuality can include all of these dimensions, not all of them are always experienced or expressed. Sexuality is influenced by the interaction of biological, psychological, social, economic, political, cultural, ethical, legal, historical, religious and spiritual factors. (n.p.)

\section{Discussion}

While the helpline presents an accessible and acceptable platform that generates useful and varied data and information from girls based on their SRH experiences, its transformative potential is inhibited by the use of pre-determined and homogeneous approaches driven by socio-cultural values and assumptions about girls' sexuality. Therefore, despite the evidence and general acknowledgement of the importance of sex education for girls, rather than using the accessibility and acceptability of the helpline by girls to address their SRH needs, the platform is used to perpetuate discourses on sexuality that rouse fear and shame, and control girls' sexuality through the discourse of sex as dangerous and girls as bad when they demonstrate sexual knowledge or acknowledge practice.

Moreover, the homogeneous approach taken by the helpline ignores the varied and complex issues raised by girls and assumes that all girls have similar needs; it fails to acknowledge that girls' sexuality experiences differ across contexts. Bhana (2007a) demonstrates the variations and complexities of children's sexuality through discussions on HIV and AIDS with white South African children from an affluent neighborhood and black South African children from economically disadvantaged backgrounds. Based on these discussions, Bhana concluded that while children had knowledge about sex and HIV and AIDS, their understandings and knowledge differed and were dependent on their sex and their experiences as well as social, cultural, and economic factors.

Given that not all cases presented to the helpline are of non-consensual sexual activity, the approach taken by CLK does not interrogate why girls are engaging in sex. While it is important for girls to understand their vulnerability and be able to protect themselves and respond appropriately to abuse, the pre-determined abstinence approach and homogeneous perspec- 
tive taken by CLK fails to acknowledge that girls are sexually active, and are so for a variety of reasons, hence the need for a more open and differentiated approach than one that simply discourages sexual activity.

Similar to many conventional sexuality education programs, CLK uses a top-down approach that is not inclusive and responsive to the articulated needs of girls, but one that is based on what is deemed fit by project staff. Therefore, while the helpline presents opportunities for child-centered interventions, with content driven by the needs of girls, the agenda here is predetermined and driven by social-cultural ideas about girls' sexuality.

\section{Conclusion}

Helplines can increase opportunities for girls to exercise agency on matters regarding their SRH needs. However, even with these technological advancements, information on SRH that is available to girls through some communication technologies can mimic that delivered through conventional means because of barriers posed by cultural and religious biases related to girls' sexuality.

We need to rethink communication technologies and how they respond to girls' SRH issues. Helplines, for example, should not only be concerned with creating spaces in which girls can present their issues, but should also interrogate the values expressed by their operators. Spaces that aim to facilitate girls' participation should be discursive in creating room for dialogue, confrontation, deliberation, and critical thinking (Moss and Petrie 2002).

Communication technology needs to be looked at differently. Beyond providing voice to girls they could be instrumental in transforming society by challenging and changing the perceptions, values, and attitudes that create socio-cultural inequalities and place constraints on the capabilities of girls.

Moreover, a contextual approach needs to be used. While boundaries between adults and children as well as gender norms have been changing over many decades in Western society, there are particular challenges in translating these to many African contexts where stronger gender and authority hierarchies exist between adults and children. Therefore, even though democratic processes and gender dynamics are shifting, they are still quite fragile in Africa. For communication technologies to be transformational in addressing girls' SRH needs, these contextual power and cultural dynamics must be understood, interrogated, and challenged. Only then can 
technologies live up to their potential of creating non-violent societies for girls and young women.

$\cos$

JOAN NJAGI is a qualitative researcher with a multi-disciplinary background in children and youth in development, public health (with a focus on reproductive health) and education. Her research interests center on understanding how innovations in sexuality education interact with contextual social norms and structural power relations to shape adolescent sexual and reproductive health rights. E-mail: wanjiranjagi@gmail.com

\section{References}

Bent, Emily. 2013. "A Different Girl Effect: Producing Political Girlhoods in the "Invest in Girls" Climate." In Youth Engagement: The Civic-Political Lives of Children and Youth (Sociological Studies of Children and Youth, Vol. 16), ed. Sandi Kawecka Nenga and Jessica K. Taft, 3-20. Bingley, UK: Emerald Group Publishing Limited.

Bhana, Deevia. 2007a. "Childhood Sexuality and Rights in the Context of HIV/AIDS, Culture, Health \& Sexuality." An International Journal for Research, Intervention and Care 9 (3): 309-324.

Bhana, Deevia. 2007b. "The Price of Innocence: Teachers, Gender, Childhood Sexuality, HIV and AIDS in Early Schooling." International Journal of Inclusive Education 11 (4): 431-444. doi: 10.1080/13603110701391394

Bhana, Deevia. 2008. "Discourses of Childhood Innocence in Primary School HIV/AIDS Education in South Africa." African Journal of AIDS Research 7 (1): 149-158. doi: 10.2989/AJAR.2008.7.1.15.443

Bordonaro, Lorenzo. 2012. "Agency Does Not Mean Freedom. Cape Verdean Street Children and the Politics of Children's Agency." Children's Geographies 10 (4): 413-426.

doi: $10.1080 / 14733285.2012 .726068$

Burkholder, Casey, Mona Makramalla, Ehaab Abdou, Nazeeha Khoja, and Fatima Khan. 2015. "Why Study Power in Digital Spaces Anyway? Considering Power and Participatory Visual Methods." Perspectives in Education 33 (4): 6-22.

Chege, Fatuma. 2007. "Researching Gender: Explorations into Sexuality and HIV/AIDS in African Contexts." Working Paper No. 7. Cambridge: RECOUP Publications.

Childline Kenya 2012. Childline Kenya November 2012 Monthly Report. Nairobi: Childline Kenya. 
Childline Kenya 2013a. Child Helpline International Data Questionnaire: 2013 data. Nairobi: Childline Kenya.

Childline Kenya 2013b. Childline Kenya September 2012 Monthly Report. Nairobi: Childline Kenya.

Childline Kenya 2014. Childline Kenya ASK Report: Reproductive Health and Right, January to June 2014. Nairobi: Childline Kenya.

Childline Kenya 2015. Child Helpline International Data Questionnaire: 2015 data. Nairobi: Childline Kenya.

Cornwall, Andrea, and Karen Brock. 2005. "What Do Buzzwords Do For Development Policy? A Critical Look at 'Participation', 'Empowerment' and 'Poverty Reduction.”' Third World Quarterly 26 (7): 1043-1060.

Hart, Jason. 2008. "Children's Participation and International Development: Attending to the Political." International Journal of Children's Rights 16: 407418. doi: $10.1163 / 157181808 X 311231$

Hart, Laurel, and Claudia Mitchell. 2015. "From Spaces of Sexual Violence to Sites of Networked Resistance: Re-imagining Mobile and Social Media Technologies." Perspectives in Education 33 (4): 135-150.

Kendall, Nancy, and Chloe O'Gara. 2007. "Vulnerable Children, Communities and Schools: Lessons from Three HIV / AIDS Affected Areas." Compare: A Journal of Comparative and International Education 37 (1): 5-21.

Mattila, Mirkka. 2011. Mobile Technologies for Child Protection; a Briefing Note. Dakar: UNICEF.

Moss, Peter, and Pat Petrie. 2002. From Children's Services to Children's Spaces: Public Policy, Children and Childhood. London: Routledge.

Njau, Bernard, Sabina Mtweve, Longin Barongo, Rachel Manongi, Juliet Chugulu, Marcelina Msuya, Stella Mwampeta, Bertha Kiwale, Joseph Lekule, and Hector Jalipa. 2007. "The Influence of Peers and Other Significant Persons on Sexuality and Condom-Use among Young Adults in Northern Tanzania." African Journal of AIDS Research 6 (1): 33-40. doi: 10.2989/ 16085900709490397

Njagi, Joan. 2014. "Between Rhetoric and Reality: Bridging Participation Gaps in Addressing the Lived Realities of Young People's Sexuality in Kenya." Unpublished MA diss., University College London.

Njue, Carolyne, Helene Voeten, and Beth Maina Ahlberg. 2011. "Youth in a Void': Sexuality, HIV/AIDS and Communication in Kenyan Public Schools." Sex Education: Sexuality, Society and Learning 11 (4): 459-470. doi: 10.1080/ 14681811.2011.595271

Pattman, Rob, and Fatuma Chege. 2003. "'Dear Diary I Saw an Angel, and She Looked Like Heaven and Earth': Sex Talk and Sex Education.” Africa Journal of AIDS Research 2 (2): 103-112.

Ruto, Sarah. 2009. "Sexual Abuse of School Age Children: Evidence from Kenya." Journal of International Cooperation in Education 12 (1): 177-192. 
Stars Foundation 2010. Childline Kenya http:/www.starsfoundation.org.uk/ awards/organisations/childline-kenya (accessed 23 March 2017).

The Women's Rights Association Programme for Progressive Communication (WRPAPC). 2012. Going Visible: Women's Rights on the internet. Addressing Inequalities, the Heart of the Post-2015 Development Agenda and the Future we want for all: UN Women, UNICEF and United Nations. https://www.apc. org/en/pubs/going-visible-women\%E2\%80\%99s-rights-internet

Van Beelen Nel. 2007. "Learning about Sex." Exchange on HIVIAIDS, Sexuality and Gender 3: 1-16.

White, Sarah, and Shyamol Choudhury. 2007. "The Politics of Child Participation in International Development: The Dilemma of Agency." European Journal of Development Research 19: 529-550. doi: 10.1080/ 09578810701667508

World Health Organization (WHO). 2006. Defining Sexual Health. Report of a Technical Consultation on Sexual Health. Geneva: World Health Organization. Young and Sexual: An Exploration of Young People's Sexuality and Their Sexual Development. 2008. Utrecht, The Netherlands: Rutgers Nisso Groep-Youth Incentives 